\title{
Maternal diabetes impairs the initiation of meiosis in murine female germ cells
}

\author{
XU-HUA QIU*, MEI-LING LI, NG LI*, QIN SUN*, JI ZHOU, RU-JUN MA, \\ YING LIN, MIN XIE, XIE GE, LI CHEN and BING YAO \\ Reproductive Medical Center, Nanjing Jinling Hospital, Nanjing University \\ School of Medicine, Nanjing, Jiangsu 210002, P.R. China
}

Received October 11, 2016; Accepted June 1, 2017

DOI: $10.3892 / \mathrm{mmr} .2017 .7245$

\begin{abstract}
Gestational diabetes mellitus (GDM) is characterized by an initial diagnosis of glucose intolerance during pregnancy. There is increasing evidence supporting the association between GDM and the inhibited development of several organs in offspring. In the present study, a murine GDM model was established in mice by intraperitoneal injection of streptozotocin to evaluate the effect of maternal diabetes on the initiation of meiosis in female germ cells of offspring. The effect of GDM on the initiation of meiosis in the offspring was evaluated by reverse transcription-quantitative polymerase chain reaction, flow cytometry and hematoxylin and eosin staining. The results showed that, compared with the control group, fetal ovary growth was inhibited, the expression levels of meiosis-specific genes, stimulated by retinoic acid gene 8 , synaptonemal complex protein, and DNA meiotic recombinase were inhibited, and the number of primordial/primary follicles was reduced in the GDM group. These may have been induced by an increase of apoptosis and inhibition of growth, as the mRNA levels of $p 21$, a vital G1 cell cycle inhibitor, and apoptotic genes were upregulated, whereas the expression levels of genes important in folliculogenesis were decreased in the GDM group. In conclusion, the data obtained in the present study suggested that maternal diabetes may impair the initiation of meiosis and ovarian growth via growth inhibition, cell cycle arrest and the induction of apoptosis.
\end{abstract}

\section{Introduction}

The initiation of oogonia into meiosis is a critical event in the reproduction of female mammals. In the embryos of

Correspondence to: Dr Bing Yao, Reproductive Medical Center, Nanjing Jinling Hospital, Nanjing University School of Medicine, 305 East Zhongshan Road, Nanjing, Jiangsu 210002, P.R. China

E-mail: yaobing@nju.edu.cn

${ }^{*}$ Contributed equally

Key words: primordial germ cell, meiosis, gestational diabetes mellitus, cell cycle, apoptosis mice, the precursors of germ cells, primordial germ cells, migrate into the gonadal ridges between 9.5 days post-coitus $(\mathrm{dpc})$ and $11.5 \mathrm{dpc}$, and their bipotential types are altered at $12.5 \mathrm{dpc}$ (1). Oogonia gradually enter into prophase of the first meiosis from $13.5 \mathrm{dpc}$ and remain arrested at the diplotene stage of meiotic prophase I until birth (2). In previous decades, a number of regulators have found to be involved in the initiation of meiosis. The stimulated by retinoic acid gene 8 (Stra8) gene is a decision gene for the transition from mitosis to meiosis in both genders (3). In embryos lacking Stra8, the oogonia fail to undergo premeiotic DNA replication, meiotic chromosome condensation, cohesion, synapsis and recombination (4).

Gestational diabetes mellitus (GDM) is a specific type of diabetes, which is characterized by the initial diagnosis of glucose intolerance during pregnancy (5). GDM is associated with high rates of birth defects and perinatal mortality. Furthermore, offspring of women with GDM are more prone to developing neonatal complications, obesity in childhood, diabetes, and hyperlipidemia in adulthood (6-8). Previous studies have indicated that GDM also adversely affects the reproductive function of the next generation. In male offspring, maternal diabetes causesdecreases in testis weight, numbers of mature spermatids and daily sperm production (9). Female offspring of diabetic rats have been shown to exhibit decreased ovary weight, follicle number and reproductive performance (10). However, the effect of GDM on the initiation of meiosis remains to be fully elucidated.

In the present study, the effect of maternal diabetes on the initiation of meiosis in female offspring was examined in order to determine the mechanism responsible for the impaired reproductive function of female offspring.

\section{Materials and methods}

Chemicals and media. All chemicals and culture media were purchased from Sigma; Merck Millipore (Darmstadt, Germany) unless stated otherwise.

Animals. A total of 60CD-1 mice (weight, 18-23 g; age, 6-8 weeks) were used in the present study (Vital River Laboratories, Beijing, China). All procedures performed in the 
animal experiments were approved by the Ethical Committee of Jinling Hospital (Nanjing, China). The mice were maintained on a $12 \mathrm{~h}$ light/dark cycle at $20^{\circ} \mathrm{C}$ with free access to water and food. Mating was timed overnight and the appearance of a vaginal plug was considered to be $0.5 \mathrm{dpc}$ in the subsequent morning.

Generation of the GDM model. To establish the GDM model (Fig. 1), 30 pregnant CD-1 mice were administered with a single injection of streptozotocin (STZ) at a dose of $190 \mathrm{mg} / \mathrm{kg}$. After 4 days, the blood glucose levels were determined from tail-blood samples. The mice with glucose levels $>300 \mathrm{mg} / \mathrm{dl}$ were considered to be a model of GDM. A total of 15 age-matched pregnant mice were injected with citrate buffer as a control.

Reverse transcription-quantitative polymerase chain reaction $(R T-q P C R)$ analysis. Ovarian tissues were isolated from fetuses at $14.5 \mathrm{dpc}$, with three litters per group. Total RNA was extracted using an RNAprep pure Micro kit (Tiangen Biotech Co., Ltd., Beijing, China) according to the manufacturer's protocol. First-strand cDNA was synthesized using PrimeScript RT master mix (Takara Bio, Inc., Shiga, Japan) and diluted to $100 \mu \mathrm{g} / \mathrm{ml}$. The mRNA levels were determined using RT-qPCR analysis in a $10 \mu \mathrm{l}$ reaction volume $(5 \mu \mathrm{l}$ SYBR premix, $1 \mu 1$ primer mix, $100 \mathrm{ng}$ cDNA, nuclease free water to $10 \mu \mathrm{l}$ ). The primers used for amplification are listed in Table I. The PCR condition was as follows: $95^{\circ} \mathrm{C}$ for $10 \mathrm{~min}$, followed by 40 cycles at $95^{\circ} \mathrm{C}$ for $10 \mathrm{sec}, 60^{\circ} \mathrm{C}$ for $30 \mathrm{sec}$ and a final cooling step at $4^{\circ} \mathrm{C}$ for $10 \mathrm{~min}$. The experiment was repeated independently three times. GAPDH was used as a housekeeping positive control. The relative fold-change in gene expression was evaluated using the $\Delta \Delta \mathrm{Cq}$ method (11). $G A P D H$ served as the endogenous control.

Follicle counting. The day of delivery was defined as postnatal day (PND) 0 . The ovaries of the mice were isolated on PND3 and fixed with $4 \%$ paraformaldehyde (nine ovaries per group), embedded in paraffin, and serially sectioned at $5 \mathrm{~mm}$. Every third section was collected for HE staining. The numbers of primordial follicles/primary follicles and secondary follicles were examined by anIX3-SVRinverted microscope (Olympus Corporation, Tokyo, Japan) and counted in a double-blinded manner. The primordial follicle was defined as a single oocyte surrounded by squamous granulosa cells. The primary follicle contained an oocyte, which was surrounded by cuboidal granulosa cells. The secondary follicle was defined as an oocyte surrounded by two or more layers of granulosa cells.

Cell cycle analysis. The ovarian tissues isolated from the offspring were digested with trypsin $(0.5 \%)$ and washed with pre-cold PBS (three litters per group). Subsequently, the cells were fixed with $70 \%$ ethanol overnight and stained with propidium iodide $(50 \mu \mathrm{g} / \mathrm{ml})$ for $30 \mathrm{~min}$. The fluorescence was then analyzed using a FACSCalibur system (Miltenyi Biotec $\mathrm{GmbH}$, Bergisch Gladbach, Germany). The experiment was repeated independently three times.

Statistical analysis. The results are expressed as the mean \pm standard error of the mean. The statistical significance of differences was determined using a Student's two-tailed t-test with SPSS software version 19.0 (IBM Corp, Armonk, NY, USA). $\mathrm{P}<0.05$ was considered to indicate a statistically significant difference.

\section{Results}

Maternal diabetes results in growth retardation of offspring. As birth weight is the primary characteristic of intrauterine growth restriction (IUGR), the present study measured the body mass of the offspring at $14.5 \mathrm{dpc}$ and PND 3. The data showed that the mean body weights of offspring of the GDM mice were decreased significantly, compared with the control offspring on $14.5 \mathrm{dpc}(0.22$, vs. $0.32 \mathrm{~g} ; \mathrm{P}<0.01)$ and PND 3 (1.72, vs. $2.42 \mathrm{~g} ; \mathrm{P}<0.01)$ as shown in Fig. $2 \mathrm{~A}$ and $\mathrm{B}$.

In order to confirm the effect of maternal diabetes on development of the ovaries, the morphologies of the ovarian tissues from offspring at 14.5 and $15.5 \mathrm{dpc}$ were also analyzed. As shown in Fig. 2C, the appearance of the ovarian tissues from the $15.5 \mathrm{dpc}$ fetus in the GDM group was similar to those from the $14.5 \mathrm{dpc}$ fetus in the control group. The section area of the ovarian tissues from the $14.5 \mathrm{dpc}$ fetus was significantly decreased in the GDM group, compared with the control group (0.236, vs. $0.266 \mathrm{~mm}^{2} ; \mathrm{P}<0.01$; Fig. 2D).

Maternal diabetes inhibits the expression of meiosis-specific genes. Stra8 has been described as a decisive gene in the transition from mitosis to meiosis. Disrupted meiotic cDNA (Dmcl) and synaptonemal complex protein 3 (sycp3) are also important for the initiation of meiosis $(12,13)$. In order to determine the possible involvement of maternal diabetes in the initiation of meiosis in the offspring, the mRNA levels of Stra8, Dmcland Sycp3 in the fetal ovarian tissues at $14.5 \mathrm{dpc}$ were measured using RT-qPCR analysis. The data showed that the mRNA levels of the three genes were significantly decreased in the GDM groups, compared with those in the control group (Fig. 3).

Maternal diabetes inhibits primordial follicle assembly. As shown in Fig. 4, morphometric analysis of the PND3 ovaries revealed a significant decrease in the number of the primordial/primary follicles in the offspring of the GDM group, compared with those of the control group, whereas the numbers of secondary follicles were significantly increased.

Maternal diabetes induces cell cycle arrest of ovarian cells of the offspring. To elucidate the mechanisms underlying the inhibited ovarian growth in the GDM group, the present study examined the role of GDM in cell cycle progression. The data showed that maternal diabetes led to the induction of G1 cell cycle arrest in the fetal ovarian tissues. The percentage of cells in the $\mathrm{S}$ phase was significantly decreased, compared with those in the control group (4.97, vs, $11.22 \%$; $\mathrm{P}<0.01$ ). Although there were no significant differences between the percentages of ovarian cells in the G1 (78.35, vs. $67.48 \%$; $\mathrm{P}=0.13)$ and $\mathrm{G} 2(16.68$, vs. $21.29 \% ; \mathrm{P}=0.40)$ phases between the two groups (Fig. 5A and B), the cells in the G1 phase were increased in the GDM group. The mRNA levels of G1 cell cycle regulatory factors involved in the $\mathrm{G} 1$ to $\mathrm{S}$ transition, including Cyclin E, cyclin-dependent kinase 2 and $p 21$, 
Table I. Primers used for reverse transcription-quantitative polymerase chain reaction analysis.

\begin{tabular}{llll}
\hline Gene & Forward primer sequence (5'-3') & Reverse primer sequence (5'-3') & Product size (bp) \\
\hline GAPDH & TCTTGCTCAGTGTCCTTGC & CTTTGTAAGCTCATTTCCTGG & 133 \\
Stras & CTCCTCCTCCACTCTGTTGC & GCGGCAGAGACAATAGGAAG & 135 \\
Sycp3 & GGGGCCGGACTGTATTACT & AGGCTGATCAACCAAAGGTG & 169 \\
Dmcl & CCCTCTGTGTGACAGCTCAAC & GGTCAGCAATGTCCCGAAG & 114 \\
Figla & GATACTCGGCTGTGTTCTGG & TGGTAGGTTGGGTAGCATTTC & 137 \\
Nobox & AAAAGACCCGAACCCTGTAC & GTTCTGAAACCACACCATGATTC & 149 \\
Lhx 8 & ACAGTTCGCTCAGGACAAC & AAGAATGGTTGGGACTGACG & 143 \\
BMP15 & TTATACCATCGTTCGGCTGAC & GAAAGTCCAGGGTCTGTACATG & 142 \\
Bcl2 & TAAGCTGTCACAGAGGGGCT & TGAAGAGTTCCTCCACCACC & 147 \\
Bax & TTGGAGATGAACTGGACAGC & CAGTTGAAGTTGCATCAGC & 148 \\
FasL & AAGTCCCAGAAATCGCCTATG & GGTATGGTTCACGACTGGAG & 120 \\
$P 21$ & GTATCAGCTCTTCCACCTGC & TGTTAAATGGGCCACACTCC & 128 \\
CDK2 & GTGAGGAGGAGCATGAATGGA & CGGAACAGGTCGGACATCAC & 152 \\
Cyclin E & GCATTCCTCTTCCCCTCATC & GGACCCCTCTGCATTGATAAG & \\
\hline
\end{tabular}

Stra8, stimulated by retinoic acid gene $8 ;$ Sycp3, synaptonemal complex protein 3; Dmc1, DNA meiotic recombinase 1; Figla, folliculogenesis-specific bHLH transcription factor; Lhx8, LIM homeobox 8; BMP15, bone morphogenetic protein 15; Bcl2, B-cell lymphoma 2; Bax, Bcl2-associated X protein; FasL, Fas ligand; CDK2, cyclin-dependent kinase 2; Nobox, NOBOX oogenesis homeobox.

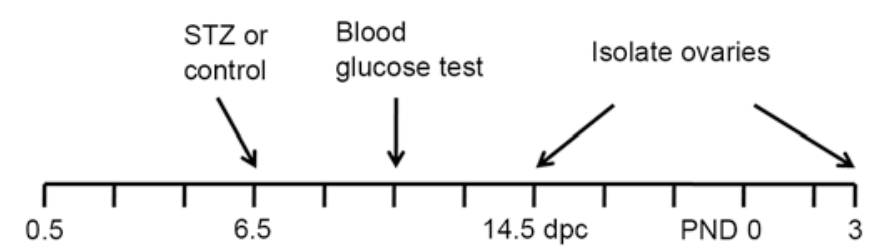

Figure 1. Schematic illustration of the timescale of GDM induction, blood glucose level assessment and ovary collection. Pregnant mice were administered with an injection of $190 \mathrm{mg} / \mathrm{kg} \mathrm{STZ}$ intraperitoneally at $6.5 \mathrm{dpc}$. After 4 days, blood glucose levels were assessed from a tail-blood sample. The mice with glucose levels of $\geq 300 \mathrm{mg} / \mathrm{dl}$ were considered a GDM model. Ovarian tissues were isolated from $14.5 \mathrm{dpc}$ embryos or PND 3 litters for the subsequent experiments. GDM, gestational diabetes mellitus; STZ, streptozotocin; dpc, days post-coitus; PND, postnatal day.

were also determined in the fetal ovarian tissues. As shown in Fig. 5C, the levels of $p 21$ were significantly increased (4.06-fold), compared with the control group, indicating G1 arrest.

Maternal diabetes is associated with increased apoptosis and inhibited growth of ovarian cells in offspring. In addition to G1 cell cycle arrest, the p21 signaling pathway is also involved in the induction of apoptosis (14). Therefore, the present study evaluated the effect of GDM on cell apoptosis. The results showed that the ratio of apoptotic genes B-cell lymphoma 2 $(\mathrm{Bcl} 2) / \mathrm{Bcl} 2$-associated $\mathrm{X}$ protein and the expression of Fas were respectively increased by 1.58 - and 1.72 -fold in the GDM group (Fig. 6A), suggesting that the apoptosis of fetal ovarian tissues was increased by GDM.

As cells in the $S$ phase were significantly reduced, the levels of growth-associated genes were also examined. It was found that the mRNA levels of four genes important in folliculogenesis, Nobox, bone morphogenetic protein 15, folliculogenesis-specific bHLH transcription factor and LIM homeobox 8, were significantly decreased (Fig. 6B).

\section{Discussion}

There is increasing evidence of the association between IUGR and cardiac diseases, hypertension, diabetes and obesity. The IUGR induced by diabetes adversely affects the development of several organs postnatally (15-17). Previous studies have also revealed that the reproductive function of offspring is impaired by maternal diabetes $(9,10)$. However, few studies have investigated the association between maternal diabetes and the initiation of meiosis in female offspring. As GDM affects $3-5 \%$ of all pregnancies in the US and $\sim 17.5 \%$ in China, the present study evaluated the impact of GDM on the initiation of meiosis in offspring $(5,18)$. In the present study, it was demonstrated that maternal diabetes inhibited the initiation of meiosis in female germ cells via cell cycle arrest, apoptosis induction and growth inhibition.

As body weight provides a general overview of health status, it is important. Low birth weight is considered to be the primary characteristic of growth retardation (19). In the present study, it was found that the body mass of offspring was significantly decreased in the GDM group, not only at PND 3 , but also at $14.5 \mathrm{dpc}$. This is consistent with the results of previous studies, which showed that low birth weight occurred frequently in STZ-induced diabetes $(9,10)$. The present study also found that the development of ovarian tissues in offspring was delayed by at least 1 day in the GDM group. These data indicated that the ovarian growth in offspring was rapidly impaired by the hyperglycemic intrauterine environment, 
A

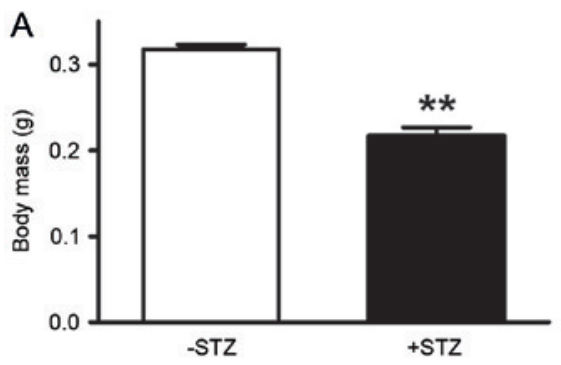

C

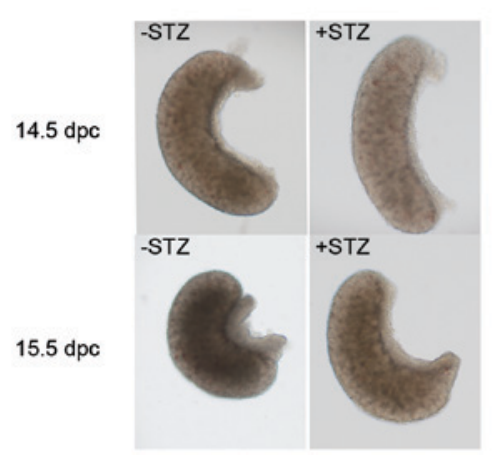

B

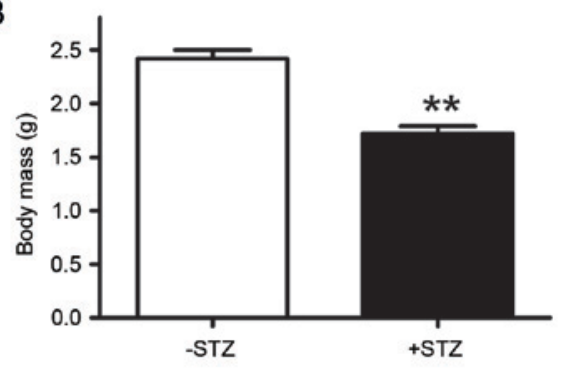

D

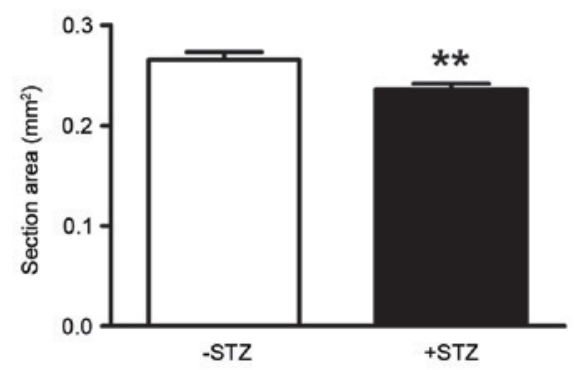

Figure 2. Maternal diabetes results in growth retardation. (A) Body mass of 14.5 dpc embryos. (B) Body mass of postnatal day 3 pups. (C) Morphologies of 14.5 and $15.5 \mathrm{dpc}$ ovarian tissues. (D) Section area of $14.5 \mathrm{dpc}$ ovarian tissues. ${ }^{* *} \mathrm{P}<0.01$ vs. control group. STZ, streptozotocin; dpc, days post-coitus.

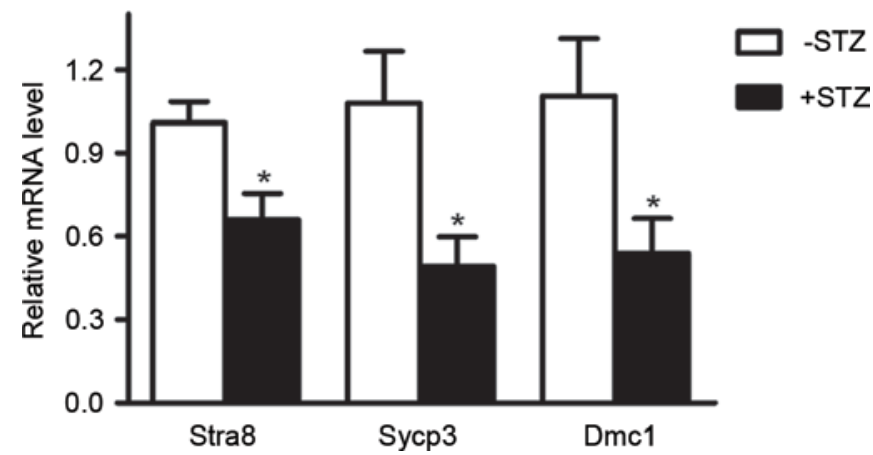

Figure 3. Maternal diabetes inhibits the expression of meiosis-specific genes. The mRNA levels of Stra8, Sycp3 and Dmcl were measured using reverse transcription-quantitative polymerase chain reaction analysis in ovarian tissues of $14.5 \mathrm{dpc}$ embryos. " $\mathrm{P}<0.05$ vs. control group. Stra 8 , stimulated by retinoic acid gene 8; Sycp3, synaptonemal complex protein 3; Dmc1, disrupted meiotic cDNA; STZ, streptozotocin; dpc, days post-coitus.

and suggested that GDM requires prompt treatment following diagnosis to avoid the rapid effects of the hyperglycemic environment on organ development.

As meiosis of the murine female germ cells was initiated at $13.5 \mathrm{dpc}$ and gradually progressed into the diplotene stage, with arrest at this stage at birth, the inhibited ovarian growth at $14.5 \mathrm{dpc}$ indicated that the meiotic initiation was possibly affected by GDM. This was confirmed by revealing that the mRNA levels of Stra 8 in fetal ovarian tissues, and of another two important meiosis-specific genes, were significantly decreased in the GDM group. Stra8, Dmcl and sycp3 are three essential genes for the initiation of meiosis $(3,12,13)$. The absence of either gene results in the inhibition of meiosis. As the normal initiation of meiosis is important for oocyte growth and folliculogenesis, the reduced expression of these genes may result in the decreased number of primordial follicles.
This was supported by the data obtained in the present study, which showed that the number of primordial/primary follicles was reduced in the GDM group, and data reported in a previous study (10). Of note, the present study found that the number of secondary follicles was increased in the GDM group, suggesting risk of early activation of the follicle pool and premature ovarian failure. In mammals, the number of primordial follicles represents the reproductive ability of a female organism. In the present study, the reduction in the numbers of primordial/primary follicles and the early activation of the follicles suggested that the reproductive function of the offspring was impaired by maternal diabetes.

In order to determine the mechanism underlying the reduced numbers of primordial/primary follicles, the present study analyzed the effect of GDM on cell cycle, apoptosis and growth. Data showed that G1 cell cycle arrest, apoptosis induction and growth retardation of the fetal ovarian cells were all induced by maternal diabetes. These results suggested that the effect of GDM on the initiation of meiosis in the female germ cells of the offspring was varied and comprehensive. P21 is an important G1 cell cycle inhibitor, which is expressed in granulosa cells of the ovary (20). The activation of the p21 signaling pathway is responsible for the apoptosis of granulosa cells and premature ovarian failure induced by Tripterygium glycosides (14). It is also involved in luteinizing hormone-regulated follicular growth arrest (21). The data obtained in the present study showed that the expression level of p21 was significantly increased (4.06-fold) in the fetal ovarian tissues of the GDM group, suggesting that activation of p21 signaling pathway may be responsible for the induction of apoptosis and growth retardation.

Taken together, the present study demonstrated that the initiation of meiosis was inhibited by maternal diabetes, possibly by cell cycle arrest, apoptosis induction and growth inhibition regulated by the p21 signaling pathway. 
A

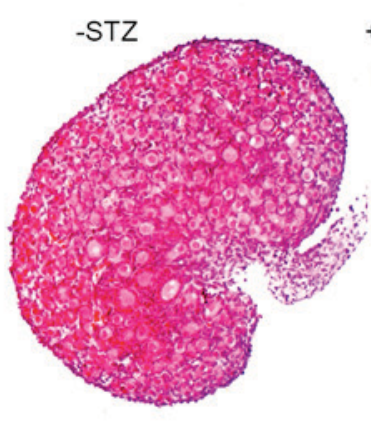

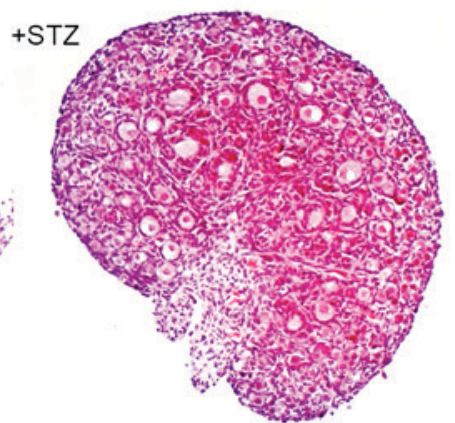

B

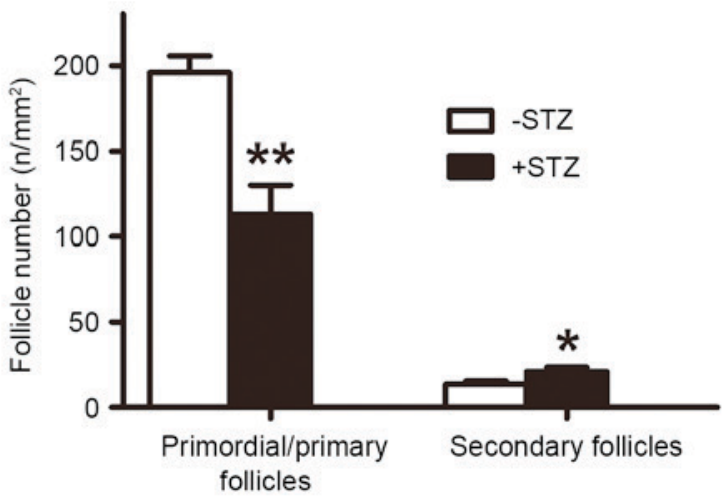

Figure 4. Maternal diabetes inhibits primordial follicle assembly. (A) Morphologies of postnatal day 3 ovarian tissues. Magnification, x100. (B) Numbers of different classes of follicles. ${ }^{*} \mathrm{P}<0.05$ and ${ }^{* *} \mathrm{P}<0.01$, vs. control group. STZ, streptozotocin.

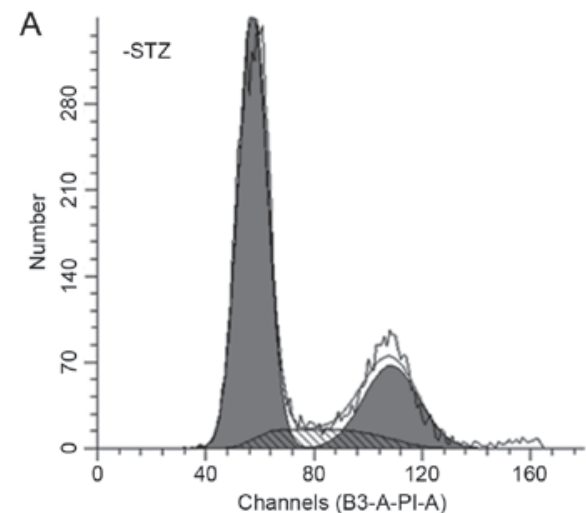

B

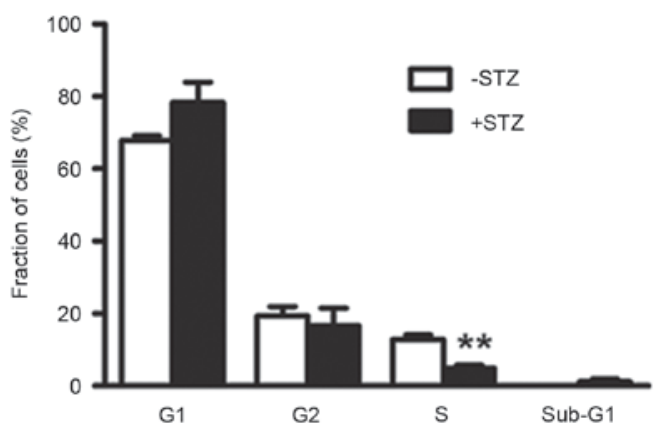

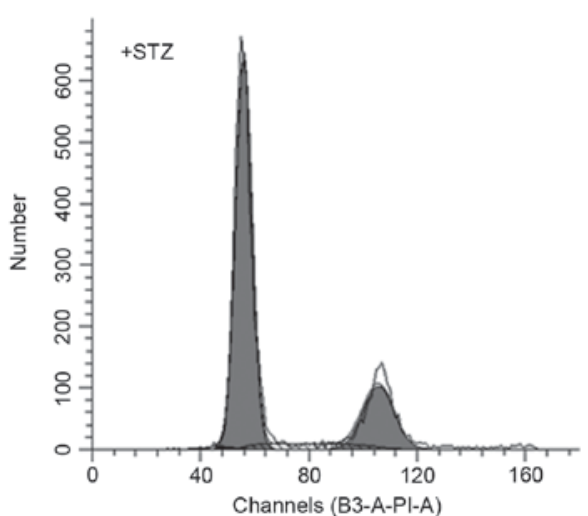

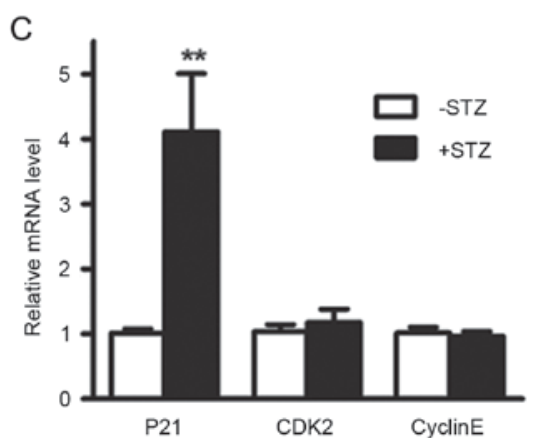

Figure 5. Maternal diabetes increases the induction ofG1 cycle arrest in ovarian tissue of offspring. (A) Cell cycle analysis of ovarian cells of embryos at 14.5 days post-coitus. (B) Quantification of the results of cell cycle analysis. (C) mRNA levels of G1 cell cycle regulators." P<0.01 vs. control group. CDK2, cyclin-dependent kinase; STZ, streptozotocin.
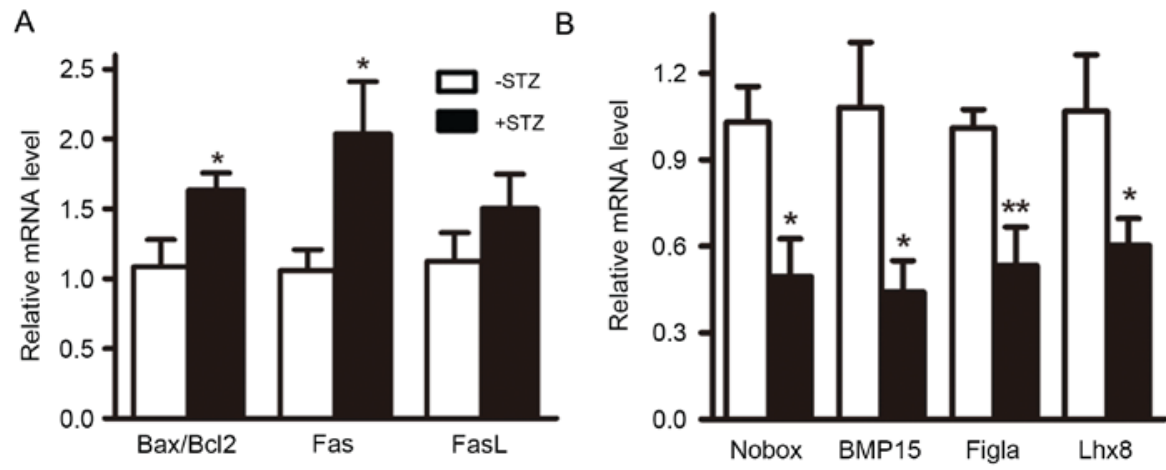

Figure 6. Maternal diabetes results in induction of apoptosis and growth inhibition. (A) mRNA levels of apoptotic genes. (B) mRNA levels of genes important for oocyte growth and folliculogenesis. " $\mathrm{P}<0.05$ and ${ }^{* *} \mathrm{P}<0.01$, vs. control group. Bcl2, B-cell lymphoma 2; Bax, Bcl2-associated X protein; FasL, Fas ligand; BMP15, bone morphogenetic protein 15; Figla, folliculogenesis-specific bHLH transcription factor; Lhx8, LIM homeobox 8; Nobox, NOBOX oogenesis homeobox. 


\section{Acknowledgements}

This study was supported by the State Key Development Program for Basic Research of China (grant no. 2013CB945200) and the National Natural Science Foundation of China (grant no. 81503655$)$.

\section{References}

1. Ginsburg M, Snow MH and McLaren A: Primordial germ cells in the mouse embryo during gastrulation. Development 110: 521-528, 1990.

2. Hunt PA and Hassold TJ: Sex matters in meiosis. Science 296: 2181-2183, 2002.

3. Tedesco M, Desimio MG, Klinger FG, De Felici M and Farini D: Minimal concentrations of retinoic acid induce stimulation by retinoic acid 8 and promote entry into meiosis in isolated pregonadal and gonadal mouse primordial germ cells. Biol Reprod 88: 145, 2013.

4. Baltus AE, Menke DB, Hu YC, Goodheart ML, Carpenter AE, de Rooij DG and Page DC: In germ cells of mouse embryonic ovaries, the decision to enter meiosis precedes premeiotic DNA replication. Nat Genet 38: 1430-1434, 2006.

5. Klein J, Charach R and Sheiner E: Treating diabetes during pregnancy. Expert Opin Pharmacother 16: 357-368, 2015.

6. Fetita LS, Sobngwi E, Serradas P, Calvo F and Gautier JF: Consequences of fetal exposure to maternal diabetes in offspring. J Clin Endocrinol Metab 91: 3718-3724, 2006.

7. Dabelea D, Mayer-Davis EJ, Lamichhane AP, D'Agostino RB Jr, Liese AD, Vehik KS, Narayan KM, Zeitler P and Hamman RF: Association of intrauterine exposure to maternal diabetes and obesity with type 2 diabetes in youth: The SEARCH Case-Control Study. Diabetes Care 31: 1422-1426, 2008.

8. Buchanan TA and Xiang AH: Gestational diabetes mellitus. J Clin Invest 115: 485-491, 2005.

9. Amorim EM, Damasceno DC, Perobelli JE, Spadotto R, Fernandez CD, Volpato GT and Kempinas WD: Short- and long-term reproductive effects of prenatal and lactational growth restriction caused by maternal diabetes in male rats. Reprod Biol Endocrinol 9: 154, 2011.

10. Spadotto R, Damasceno DC, Godinho AF, Amorim EM, Perobelli JE and Kempinas Wde G: Reproductive physiology, and physical and sexual development of female offspring born to diabetic dams. Arq Bras Endocrinol Metabol 56: 96-103, 2012.
11. Kongpan T, Mahasirimongkol S, Konyoung P, Kanjanawart S, Chumworathayi P, Wichukchinda N, Kidkeukarun R, Preechakul S, Khunarkornsiri U, Bamrungram W, et al: Candidate HLA genes for prediction of co-trimoxazole-induced severe cutaneous reactions. Pharmacogenet Genomics 25: 402-411, 2015.

12. Lin Y, Gill ME, Koubova J and Page DC: Germ cell-intrinsic and -extrinsic factors govern meiotic initiation in mouse embryos. Science 322: 1685-1687, 2008.

13. Reynolds N, Collier B, Bingham V, Gray NK and Cooke HJ: Translation of the synaptonemal complex component Sycp3 is enhanced in vivo by the germ cell specific regulator Dazl. RNA 13: 974-981, 2007.

14. Liu TE, Zhang L, Wang S, Chen C and Zheng J: Tripterygium glycosides induce premature ovarian failure in rats by promoting p53 phosphorylation and activating the serine/threonine kinase 11-p53-p21 signaling pathway. Exp Ther Med 10: 12-18, 2015.

15. de Bruin JP, Dorland M, Bruinse HW, Spliet W, Nikkels PG and Te Velde ER: Fetal growth retardation as a cause of impaired ovarian development. Early Hum Dev 51: 39-46, 1998.

16. Murdoch WJ, Van Kirk EA, Vonnahme KA and Ford SP: Ovarian responses to undernutrition in pregnant ewes, USA. Reprod Biol Endocrinol 1: 6, 2003.

17. Ibáñez L, Jaramillo A, Enríquez G, Miró E, López-Bermejo A, Dunger D and de Zegher F: Polycystic ovaries after precocious pubarche: Relation to prenatal growth. Hum Reprod 22: 395-400, 2007.

18. Yan J, Li X, Su R, Zhang K and Yang H: Long-term effects of maternal diabetes on blood pressure and renal function in rat male offspring. PloS One 9: e88269, 2014.

19. Chahoud I and Paumgartten FJ: Influence of litter size on the postnatal growth of rat pups: Is there a rationale for litter-size standardization in toxicity studies? Environ Res 109: 1021-1027, 2009.

20. Hu YC, Wang PH, Yeh S, Wang RS, Xie C, Xu Q, Zhou X, Chao HT, Tsai MY and Chang C: Subfertility and defective folliculogenesis in female mice lacking androgen receptor. Proc Natl Acad Sci USA 101: 11209-11214, 2004.

21. Robker RL and Richards JS: Hormone-induced proliferation and differentiation of granulosa cells: A coordinated balance of the cell cycle regulators cyclin D2 and p27Kip1. Mol Endocrinol 12: 924-940, 1998. 